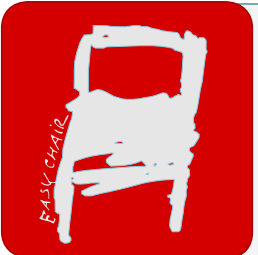

EPiC Series in Health Sciences

Volume 3, 2019, Pages 189-192

CAOS 2019. The 19th Annual Meeting of the International Society for Computer Assisted Orthopaedic Surgery

\title{
Long term Patient Outcomes following Navigated Knee Replacement: A Retrospective analysis
}

\author{
Jeldi A.J., Holloway N., Ewen A.M., Picard F. \\ Golden Jubilee National Hospital, Clydebank U.K \\ artaban.jeldi@gjnh.scot.nhs.uk
}

\begin{abstract}
Total knee replacements (TKR) are often deemed successful when patients recover and maintain their functionality both in the short and long term. The other main indicator for success is the revision rate following TKR. This study analyses the long term results of navigated TKR based on patient related outcomes in terms of patient satisfaction, Oxford Knee Score (OKS) and also knee revision rates.

The retrospective data of all patients who underwent navigated Columbus TKR from the author's institution from 2005 to 2011 was analysed and compared to the national database. The overall cohort size was 1679 with a mean age of $68.81 \mathrm{yrs}$ (sd 8.46). The OKS, satisfaction scores, complication and revision rates were compiled and evaluated up to ten years post-operatively. Patient reported outcomes were recorded six weeks, one, two, five and ten years post-operatively.

The results from the OKS and satisfaction scores demonstrated marked improvement throughout recovery to the ten year time point. The OKS had a mean improvement of 16.6 from the pre-operative score at the 10 year time point and the satisfaction scores improved at each follow-up and remained high at the 10 year time point. A total of 61 complications and 36 revisions recorded, with infection being the major cause for revisions.

This survey has identified and established that the majority of patients undergoing knee arthoplasty using the Columbus total knee system and computer navigation function reasonably well in the long term and the revision and complication rates in this hospital are compare well with national levels..
\end{abstract}

\section{Introduction}

A successful outcome of total knee replacement (TKR) surgery, which is considered the gold standard (Pozzi et al., 2014) for the treatment of osteoarthritis (OA) is often characterised by revision rates and functional recovery of patients (Patil et al., 2015). Recovery of patients is often established 
Long term Patient Outcomes following Navigated Knee Replacement:A Retrospective ...A. Jeldi et al.

by evaluating subjective, objective and functional outcomes, both at the short term and long term follow-up. It has been well established that the objective outcomes do not always correlate with the subjective outcomes in terms of recovery. Patient reported subjective outcomes need to be given additional importance when evaluating long term success of TKR (Bullens et al., 2001, Choi \& Ra, 2016). This study analyses the long term recovery of navigated TKR based on patient related outcomes in terms of patient satisfaction, Oxford Knee Scores (OKS) and knee revision rates.

\section{Methods}

All relevant clinical information was extracted from our local clinical database and national database for patients who underwent navigated TKR using the Columbus knee system (Aesculap AG, Tuttlingen, Germany) and the OrthoPilot navigation system (Aesculap AG, Tuttlingen, Germany) between 2005 and 2011. The cohort size was 1679 patients (735 Males and 944 females, mean age $68.81 \mathrm{yrs}$ (Table 1)) including 32 bilateral cases (19 males and 13 females). The essential demographics are detailed in Table 1. The OKS and satisfaction scores (Table 2) were recorded at the six weeks, one, two, five and ten years post-operatively. The OKS was scored using the 12-60 (best to worst respectively) scoring system. Satisfaction levels were measured using a 5-point Likert scale ranging from "very satisfied" to "very dissatisfied". Complications and revisions were identified using both the internal and external databases (Table 2).

\section{Results}

The pre-operative OKS had a mean of 41.8 and showed marked improvements at all time points up to ten years post-operatively. The mean difference at 10 years OKS was at 16.6 from the preoperative score. Satisfaction levels increased at each follow-up compared to that at six weeks postoperatively and remained high. One patient died within 90 days of surgery. There were a total of 65 complications and 36 revisions $(2.1 \%)$ recorded, with infection $(1.5 \%)$ being the major cause for revisions. All infected cases lead to the implant being revised, with the exception of one where the patient died of unrelated causes prior to any planned revision. Eighty-four percent $(n=21)$ of infections occurred within 90 days of surgery. 
Long term Patient Outcomes following Navigated Knee Replacement:A Retrospective ...A. Jeldi et al.

\begin{tabular}{|c|c|c|c|c|c|c|}
\hline \multicolumn{2}{|c|}{ Demographics } & Age(years) & $B M I(\mathrm{~kg} / \mathrm{m} 2)$ & ASA Score & & \\
\hline \multicolumn{2}{|l|}{ Mean(SD) } & $68.8(8.64)$ & $32.5(5.81)$ & $2.2(0.55)$ & & \\
\hline Oxford Knee Score ( 12-60) & pre-OKS & $6 \mathrm{Wk} O K S$ & $1 \mathrm{Yr} \mathrm{OKS}$ & $2 \mathrm{Yr} \mathrm{OKS}$ & $5 \mathrm{Yr} O \mathrm{OKS}$ & $10 \mathrm{Yr}$ OKS \\
\hline Mean(SD) & $41.8(7.7)$ & $27.7(7.5)$ & $22.3(7.9)$ & $22.3(8.2)$ & $21.8(8.9)$ & $25.2(10.6)$ \\
\hline \multicolumn{2}{|c|}{ Satisfaction } & $\begin{array}{l}6 W k \\
\text { Satisfaction }\end{array}$ & $\begin{array}{l}1 \mathrm{Yr} \\
\text { Satisfaction }\end{array}$ & \begin{tabular}{|l}
$2 \mathrm{Yr}$ \\
Satisfaction
\end{tabular} & \begin{tabular}{|l}
$5 \mathrm{Yr}$ \\
Satisfaction
\end{tabular} & $\begin{array}{l}10 \mathrm{Yr} \\
\text { Satisfaction }\end{array}$ \\
\hline \multicolumn{2}{|c|}{ Very Satisfied } & $67.8 \%$ & $81.5 \%$ & $81.0 \%$ & $84.7 \%$ & $84.4 \%$ \\
\hline \multicolumn{2}{|c|}{ Satisfied } & $17.3 \%$ & $12.6 \%$ & $13.3 \%$ & $9.8 \%$ & $7.5 \%$ \\
\hline \multicolumn{2}{|l|}{ Unsure } & $5.0 \%$ & $3.9 \%$ & $4.0 \%$ & $3.6 \%$ & $2.7 \%$ \\
\hline \multicolumn{2}{|l|}{ Dissatisfied } & $0.8 \%$ & $1.9 \%$ & $1.7 \%$ & $1.9 \%$ & $5.4 \%$ \\
\hline \multicolumn{2}{|c|}{ Very Dissatisfied } & $0 \%$ & $0 \%$ & $0 \%$ & $0 \%$ & $0 \%$ \\
\hline \multicolumn{2}{|c|}{ Completed follow-ups } & $93 \%$ & $85 \%$ & $28 \%$ & $32 \%$ & $11 \%$ \\
\hline \multicolumn{6}{|c|}{ Total Patients: 1679} & \\
\hline
\end{tabular}

Table 1: Patient demographics and Oxford Knee Scores and satisfaction rates up to five years post-operatively.

\begin{tabular}{|l|c|l|c|}
\hline \multicolumn{2}{|c|}{ Revisons } & \multicolumn{2}{c|}{ Complications } \\
\hline Infections* & 24 & Infections* & $21(4)$ \\
\hline Fracture & 2 & DVT/PE & 15 \\
\hline Arthrofibrosis & 2 & Mortality & 1 \\
\hline Aseptic Loosening & 2 & Stroke & 4 \\
\hline Pain & 4 & AMI & 4 \\
\hline PCL rupture trauma & 1 & GI Bleed & 2 \\
\hline Surgical Mis takes & 1 & $\begin{array}{l}\text { Acute Renal } \\
\text { Failure }\end{array}$ & 10 \\
\hline Total & $\mathbf{3 6}$ & Total & $\mathbf{6 5}$ \\
\hline
\end{tabular}

*Revision infections are a subset of complication infections.

Table 2: Revision and complication details. Complication infections are within 90 days (over 90 days).

\section{Discussion}

This retrospective review of data provides an insight in to the recovery of patients post surgery for a period of ten years. The scores achieved in both OKS and satisfaction levels suggest that most of the recovery and improvements in quality of life is obtained within the first year of surgery with continued improvements in the long term. A systematic review published by Kallenberg et al. (2018) concluded that the majority of studies report satisfaction rates above $80 \%$, with post-operative function and pain relief being primary indicators of satisfaction which are in line with the findings of this study. The routine follow-ups in the hospital were initially planned to span over a period of ten 
Long term Patient Outcomes following Navigated Knee Replacement:A Retrospective ...A. Jeldi et al.

years but were later reduced to six weeks and one year, hence the return of patients at two years $(28 \%)$, five years $(32 \%)$ and ten years $(11 \%)$ post-operatively shows a reduction, however it was clear from the national database that revisions and complications for this cohort were minimal and well below national averages and it is safe to assume that patients are doing reasonably well based on the national averages. The five year revision rates as per the Scottish Arthoplasty Project was $2.8 \%$ for knees in 2010 (SAP, 2010) which is higher than this hospital's revision rates (2.1\%) and is still lower than the current national five year rates $(2.5 \%)$ (SAP, 2018). When excluding revision for infection, the revision rate related to surgical technique is $0.7 \%$. The incidence of infection during the years between 2005-2010 was 1.4\% nationally (SAP, 2012) which is similar to the rate for this hospital $(1.5 \%)$. This survey has identified and established that for the majority of patients undergoing knee arthoplasty using the Columbus total knee system and computer navigation are functioning reasonably well in the long term and the revision and complication rates in this hospital are within the national levels.

\section{References}

Bullens PH, van Loon CJ, de Waal Malefijt MC, et al., 2001, Patient satisfaction after total knee arthroplasty: A comparison between subjective and objective outcome assessments. Sept;16(6):740-7.

Choi YC and Ra JH. 2016 , Patient Satisfaction after Total Knee Arthroplasty. Knee Surgery0 Related Research, Mar; 28(1): 1-15.

Kahlenberg CA, Nwachukwu BU, McLawhorn,AS, Cross MD, Cornell CN Padgett ED, 2018 , Patient Satisfaction After Total Knee Replacement: A Systematic Review HSS J. 2018 Jul; 14(2): 192-201.

Patil, S., McCauley, J., Pulido, P. and Colwell, C.,2015, How Do Knee Implants Perform Past the Second Decade? 19 to 25-year Follow-up of the Press-fit Condylar Design TKA. Clinical Orthopaedics and Related Research, Jan; 473(1): 135-140.

Pozzi .F, L. Snyder-Mackler.L and Zeni J., 2013, Physical exercise after knee arthroplasty: A Systematic Review of Controlled trials. EJPMR, Dec; 49(6): 877-892.

Scottish Arthoplasty Project. Annual Reports 2010, 2012, 2018 [online] Available at: https://www.arthro.scot.nhs.uk/Reports/Previous.html [Accessed 29 Jan. 2019]. 